\title{
Quantum rings of arbitrary shape and non-uniform width in a threading magnetic field
}

\author{
A. Bruno-Alfonso ${ }^{1, *}$ and A. Latgé ${ }^{2, \dagger}$ \\ ${ }^{1}$ Departamento de Matemática, Faculdade de Ciências, UNESP, Universidade Estadual Paulista, \\ Avenida Luiz Edmundo Carrijo Coube 14-01, 17033-360 Bauru, São Paulo, Brazil \\ ${ }^{2}$ Instituto de Física, Universidade Federal Fluminense, 24210-346 Niterói, Rio de Janeiro, Brazil
}

(Received 14 September 2007; revised manuscript received 7 April 2008; published 2 May 2008)

\begin{abstract}
The electronic states of quantum rings with centerlines of arbitrary shape and non-uniform width in a threading magnetic field are calculated. The solutions of the Schrödinger equation with Dirichlet boundary conditions are obtained by a variational separation of variables in curvilinear coordinates. We obtain a width profile that compensates for the main effects of the curvature variations in the centerline. Numerical results are shown for circular, elliptical, and limaçon-shaped quantum rings. We also show that smooth and tiny variations in the width may strongly affect the Aharonov-Bohm oscillations.
\end{abstract}

DOI: 10.1103/PhysRevB.77.205303

PACS number(s): 73.21.-b, 73.23.-b, 73.63.-b

\section{INTRODUCTION}

Quantum rings (QRs) are promising quantum structures because of their particular topology, which leads to the Aharonov-Bohm effect. ${ }^{1}$ This interference phenomenon is associated with persistent currents and magnetization, and its strength can be modulated by tailoring the shape and the size of the QR. ${ }^{2}$ In particular, semiconductor QRs have been the subject of intense experimental ${ }^{3-9}$ and theoretical ${ }^{10-17}$ works.

The simplest model of a quantum ring has a circular shape and uniform width. However, distorted rings must be considered because imperfections occur in growth and fabrication processes. ${ }^{3,6}$ Moreover, distorted rings have interesting electronic spectra in a threading magnetic field, with anticrossings and quenched oscillation of levels as a function of the field strength. This occurs in elliptic QRs, ${ }^{18,19}$ in circular rings with varying widths in the presence of an in-plane electric field, ${ }^{20,21}$ and in QRs with structural distortions. ${ }^{22}$

To gain a deeper understanding of the effects of ring distortions on the electronic spectrum, researchers have investigated rings of arbitrary shape..$^{23-25}$ Of course, such work involves the basic concepts of differential geometry. Electronic states in rings with a uniform width in the absence of magnetic fields were calculated. ${ }^{26,27}$ It was shown that regions with a larger curvature are more favorable for the electrons. Recently, we calculated the electronic states of thin QRs with arbitrary (but smooth) variations in curvature and width and reported numerical results for elliptical rings. ${ }^{13}$ Since the thinner regions of a ring are less favorable for the electron, we were able to obtain a width profile that compensates for the effects of the non-uniform curvature of the ellipse.

Rings of arbitrary shape in the presence of a threading magnetic field have also been considered. Namely, Pershin and Piermarocchi ${ }^{28}$ calculated persistent and radiationinduced currents in QRs of arbitrary shape, uniform width, and finite-barrier transversal confinement. The calculation of the electronic states was performed by an approximate separation of the longitudinal and transversal motions. This led to an effective longitudinal equation that contains the traditional effective potential due to the curvature profile. ${ }^{26,27}$ Within this approach, it was shown that curvature variations produce level anticrossings and flattening of the lower levels.
Similar effects were obtained in rings with impurities ${ }^{29}$ and rings lacking circular symmetry. ${ }^{9,21}$

In the present work, the electronic states in QRs of arbitrary shape threaded by a magnetic field are investigated. However, contrasting Ref. 28, the ring width is non-uniform and the transversal confinement is produced by infinite barriers. Our main motivation is to predict the combined effect of width and curvature profiles. Noncircular rings may be intentionally or nonintentionally produced. In both cases, an accurate knowledge of the width profile is needed for an appropriate calculation of the electronic states. A onedimensional equation is obtained by an appropriate separation of variables in curvilinear coordinates. The energy spectra as a function of the magnetic flux threading circular, elliptical, and limaçon-shaped rings are calculated and analyzed. We highlight interesting effects of the width nonuniformity that may be relevant for the quantum mechanics in curved spaces, ${ }^{23-25}$ for experimental studies of quantum rings, ${ }^{6,9}$ and for electronic and matter transmissions through curved waveguides. ${ }^{30-33}$ Of course, our ring model is rather simple. The boundary conditions should be improved, aiming at an accurate quantitative prediction of experimental results.

The remaining part of the paper is organized as follows: In Sec. II, we set up the problem and introduce a change from Cartesian to longitudinal and transversal coordinates. The approximate separation of variables is performed, within a variational approach, in Sec. III. The periodicity of the energy levels is demonstrated in Sec. IV, and the compensation of the effects of the width and curvature is predicted in Sec. V. Finally, the numerical results and discussions and the main conclusions are presented in Secs. VI and VII, respectively.

\section{TWO-DIMENSIONAL PROBLEM}

We calculate the states of an electron in a closed plane waveguide in a perpendicular magnetic field. This model is used to describe GaAs quantum rings, when in-plane and perpendicular motions can be separated. The vector potential of the magnetic field is taken as $\boldsymbol{A}=(-y, x, 0) B / 2$ and the stationary states satisfy 


$$
H_{x y} \Psi(x, y)=E_{2 \mathrm{D}} \Psi(x, y),
$$

where

$$
\begin{aligned}
H_{x y}= & \frac{(-i \hbar \nabla-e \boldsymbol{A})^{2}}{2 m^{*}}=\frac{\hbar^{2}}{2 m^{*}}\left[-\left(\frac{\partial^{2}}{\partial x^{2}}+\frac{\partial^{2}}{\partial y^{2}}\right)\right. \\
& \left.-\frac{i}{\lambda^{2}}\left(-y \frac{\partial}{\partial x}+x \frac{\partial}{\partial y}\right)+\frac{x^{2}+y^{2}}{4 \lambda^{4}}\right],
\end{aligned}
$$

where $m^{*}$ is the electron effective mass and $\lambda=\sqrt{\hbar /(e B)}$ is the cyclotron radius. Since the particle is confined in the ring, $\Psi(x, y)$ obeys Dirichlet boundary conditions.

In this work, the overall shape of each quantum ring is determined by a smooth closed curve, which is called the centerline of the ring. In terms of its arc length $s$, such a curve is given by the following vector equation:

$$
\boldsymbol{r}=\boldsymbol{r}_{c}(s)=x_{c}(s) \boldsymbol{e}_{x}+y_{c}(s) \boldsymbol{e}_{y},
$$

where $0 \leq s \leq L$ and $L$ is the centerline perimeter. The ring occupies the two-dimensional region swept out by a moving line segment with a variable length. The motion of the segment obeys the following conditions: (i) its midpoint describes the centerline counterclockwise, (ii) it perpendicularly intersects the centerline, (iii) its length $w$ smoothly depends on the arc length $s$ covered by the midpoint and gives the local width of the ring, and (iv) different segments do not intersect. This way, rings with non-uniform widths are easily designed.

At each point of the centerline, we define the tangent unit vector

$$
\boldsymbol{T}_{c}(s)=\frac{d \boldsymbol{r}_{c}}{d s}(s)=\dot{x}_{c}(s) \boldsymbol{e}_{x}+\dot{y}_{c}(s) \boldsymbol{e}_{y}
$$

and the normal unit vector

$$
\boldsymbol{N}_{c}(s)=\boldsymbol{e}_{z} \times \boldsymbol{T}_{c}(s)=-\dot{y}_{c}(s) \boldsymbol{e}_{x}+\dot{x}_{c}(s) \boldsymbol{e}_{y} .
$$

Here, each dot over a variable means an ordinary differentiation in the variable $s$. On the other hand, the signed curvature is defined by

$$
\frac{d \boldsymbol{T}_{c}}{d s}(s)=k(s) \boldsymbol{N}_{c}(s) .
$$

Therefore, the curvature satisfies the following equations:

$$
\begin{gathered}
\ddot{x}_{c}(s)=-k(s) \dot{y}_{c}(s), \\
\ddot{y}_{c}(s)=k(s) \dot{x}_{c}(s),
\end{gathered}
$$

and

$$
k(s)=-\dot{y}_{c}(s) \ddot{x}_{c}(s)+\dot{x}_{c}(s), \ddot{y}_{c}(s) .
$$

Regarding the geometrical characterization of the centerline, we also consider the distance $\rho(s)$ of each point to the origin of coordinates, and the area $\sigma(s)$ that the vector $\boldsymbol{r}_{c}(\bar{s})$ sweeps as the arc length $\bar{s}$ increases from 0 to $s$. Those measures are given by

$$
\rho(s)=\left|\boldsymbol{r}_{c}(s)\right|=\sqrt{x_{c}^{2}(s)+y_{c}^{2}(s)}
$$

and

$$
\sigma(s)=\frac{1}{2} \int_{0}^{s}\left[-y_{c}(\bar{s}) \dot{x}_{c}(\bar{s})+x_{c}(\bar{s}) \dot{y}_{c}(\bar{s})\right] d \bar{s} .
$$

Hence, the area enclosed by the the centerline is $S=\sigma(L)$ and the magnetic flux across this area is

$$
\Phi=\frac{S \Phi_{0}}{2 \pi \lambda^{2}},
$$

where $\Phi_{0}=h / e$ is the flux quantum.

To calculate the wave function $\Psi(x, y)$, we introduce the curvilinear coordinates $u$ and $s$. For this, we remind that the region of the ring is generated by a moving line segment, whose position, direction, and length depend on the parameter $s$. Namely, its midpoint is at $\boldsymbol{r}_{c}(s)$, it is parallel to the normal unit vector $\boldsymbol{N}_{c}(s)$, and its length is $w(s)$. Hence, the position $\boldsymbol{r}=x \boldsymbol{e}_{x}+y \boldsymbol{e}_{y}$ of an arbitrary point of the segment obeys $\boldsymbol{r}-\boldsymbol{r}_{c}(s)=-u w(s) \boldsymbol{N}_{c}(s)$, where $-1 / 2 \leq u \leq 1 / 2$. Since $N_{c}(s)$ points to the inner region of the centerline, $u=-1 / 2$ corresponds to the inner boundary of the ring and $u=1 / 2$ corresponds to the outer one.

The transformation from curvilinear to Cartesian coordinates is given by

$$
\boldsymbol{r}=\boldsymbol{r}_{c}(s)-u w(s) \boldsymbol{N}_{c}(s),
$$

where $-1 / 2 \leq u \leq 1 / 2$ and $0 \leq s \leq L$. Then, the Jacobian of the $(u, s)$ to $(x, y)$ mapping is given by

$$
J(u, s)=w(s)[1+\alpha(s) u],
$$

where $\alpha(s)=w(s) k(s)$. This determinant should be positive when $-1 / 2 \leq u \leq 1 / 2$ and $0 \leq s \leq L$. Otherwise, the boundaries of the ring would not be well defined. ${ }^{27}$ Hence, $|\alpha(s)|$ $<2$ should apply for $0 \leq s \leq L$, leading to the condition $w(s) / 2<1 /|k(s)|$ at each point of the centerline. Namely, the half-width of the ring should be smaller than the curvature radius of the centerline.

In the new variables $(u, s)$, the problem is simplified because the corresponding domain is a rectangular strip. To write the differential equation (1) in the variables $(u, s)$, we introduce the function $f(u, s)$ such that

$$
\Psi(x, y)=\frac{e^{i \chi(u, s)}}{\sqrt{J(u, s)}} f(u, s),
$$

where

$$
\chi(u, s)=\frac{e}{\hbar} \int_{0}^{u} \boldsymbol{A}(\bar{u}, s) \cdot \boldsymbol{N}_{c}(s) w(s) d \bar{u}=\frac{w(s) \rho(s) \dot{\rho}(s) u}{2 \lambda^{2}}
$$

is a phase introduced to enhance the accuracy of the variational separation of variables performed below. ${ }^{28}$ It will also allow a simple analysis of the periodicity of the AharonovBohm oscillations and their invariance under translations and rotations of the ring. Moreover, the probability density obeys

$$
|\Psi(x, y)|^{2} d x d y=|f(u, s)|^{2} d u d s .
$$

Equation (1) is transformed to 


$$
H_{u s} f(u, s)=E_{2 \mathrm{D}} f(u, s),
$$

with

$$
\begin{aligned}
H_{u s}= & \frac{\hbar^{2}}{2 m^{*}}\left[a_{20} \frac{\partial^{2}}{\partial u^{2}}+a_{11} \frac{\partial^{2}}{\partial s \partial u}+a_{02} \frac{\partial^{2}}{\partial s^{2}}\right. \\
& \left.+a_{10} \frac{\partial}{\partial u}+a_{01} \frac{\partial}{\partial s}+a_{00}\right] .
\end{aligned}
$$

By omitting the arguments $s$ and $u$ and introducing $\eta=J / w$ $=1+\alpha u$, the coefficients are

$$
\begin{gathered}
a_{20}=-\frac{1}{w^{2}}-\frac{\dot{w}^{2} u^{2}}{w^{2} \eta^{2}}, \\
a_{11}=\frac{2 \dot{w} u}{w \eta^{2}}, \quad a_{02}=-\frac{1}{\eta^{2}}, \\
a_{10}=\frac{\left(w \ddot{w}-3 \dot{w}^{2}\right) u}{w^{2} \eta^{2}}-\frac{2 \dot{w} \dot{k} u^{2}}{\eta^{3}}+\frac{i}{\lambda^{2}}\left(\frac{u^{2} \dot{w}(1+\eta)}{\eta^{2}}+\frac{2 u \dot{w} \dot{\sigma}}{w \eta^{2}}\right), \\
a_{01}=\frac{2 u w \dot{k}}{\eta^{3}}+\frac{\dot{w}}{w \eta^{2}}-\frac{i}{\lambda^{2}}\left(\frac{u w(1+\eta)}{\eta^{2}}+\frac{2 \dot{\sigma}}{\eta^{2}}\right),
\end{gathered}
$$

and

$$
\begin{aligned}
a_{00}= & -\frac{k^{2}}{4 \eta^{2}}-\frac{3 \dot{w}^{2}}{4 w^{2} \eta^{2}}+\frac{\ddot{w}}{2 w \eta^{2}}-\frac{u \dot{k} \dot{w}}{\eta^{3}}+\frac{u w \ddot{k}}{2 \eta^{3}}-\frac{5 u^{2} w^{2} \dot{k}^{2}}{4 \eta^{4}} \\
& +\frac{i}{\lambda^{2}}\left(-\frac{k \rho \dot{\rho}}{2 \eta^{2}}+\frac{\dot{w} \dot{\sigma}}{w \eta^{2}}+\frac{u \dot{w}(1+\eta)}{2 \eta^{2}}+\frac{2 u w \dot{k} \dot{\sigma}}{\eta^{3}}\right. \\
& \left.+\frac{u^{2} w^{2} \dot{k}(2+\eta)}{2 \eta^{3}}\right)+\frac{1}{\lambda^{4}}\left(\frac{\dot{\sigma}^{2}}{\eta^{2}}+\frac{u w \dot{\sigma}}{\eta^{2}}+\frac{u w \dot{\sigma}}{\eta}\right. \\
& \left.+\frac{u^{2} w^{2}(1+\eta)^{2}}{4 \eta^{2}}\right) .
\end{aligned}
$$

\section{VARIATIONAL SEPARATION OF VARIABLES}

We assume that the width of the ring is much smaller than the cyclotron radius, i.e., $w \ll 2 \lambda$. Hence, for an electron in the magnetic field, the ring is essentially a one-dimensional object. We also suppose that the ring is thin enough, so that according to the Dirichlet boundary conditions, the dependence of $f(u, s)$ on the transversal coordinate $u$ may be approximated by a function of the following form:

$$
h_{n}(u)=\sqrt{2} \sin \left[n \pi\left(u+\frac{1}{2}\right)\right] .
$$

This is a stationary state with an energy $\hbar^{2} n^{2} \pi^{2} /\left(2 m^{*} w^{2}\right)$ in an infinite quantum well with a width $w$. Accordingly, the two-dimensional wave function is written as

$$
f_{n}(u, s)=h_{n}(u) g_{n}(s),
$$

where $g_{n}(s)$ is a longitudinal mode to be determined. This approximation was already used by Pershin and Piermarocchi. ${ }^{28}$

It is worth noting that Eq. (26) represents a kind of adiabatic approximation, where $f(u, s)$ is separated into a transversal mode $h_{n}(u)$ and a longitudinal mode $g_{n}(s)$. However, regarding $\Psi(x, y)$, one should bear in mind that the factor $\chi(u, s)$ in Eq. (15) admixes the transversal and longitudinal motions. This mixing is due to the magnetic field.

The longitudinal modes $g_{n}(s)$ should minimize the mean value of $H_{u s}$. Then, they are obtained through a variational calculation. By taking the normalization condition $\left\langle f_{n} \mid f_{n}\right\rangle_{u s}$ $=\left\langle g_{n} \mid g_{n}\right\rangle_{s}=1$ into account, one looks for the functions $g_{n}(s)$, which make the variation in the following functional:

$$
L_{n}\left(g_{n}\right)=\left\langle f_{n}\left|H_{u s}-E_{2 \mathrm{D}}\right| f_{n}\right\rangle_{u s}
$$

vanish. This is a necessary condition for the minimization of $L_{n}\left(g_{n}\right)$ and leads to an ordinary differential equation for $g_{n}(s)$ with the periodic boundary conditions $g_{n}(L)=g_{n}(0)$ and $\dot{g}_{n}(L)=\dot{g}_{n}(0)$. The indices below the bracket \langle\rangle define the integration variables.

For an arbitrary variation $\delta g_{n}$, the variation in the functional is

$$
\delta L_{n}=\left\langle g_{n}(s)\left|\Omega_{n}\right| \delta g_{n}(s)\right\rangle_{s}+\left\langle\delta g_{n}(s)\left|\Omega_{n}\right| g_{n}(s)\right\rangle_{s},
$$

where

$$
\Omega_{n}=\left\langle h_{n}(u)\left|H_{u s}\right| h_{n}(u)\right\rangle_{u}-E_{2 \mathrm{D}}=\frac{\hbar^{2}}{2 m^{*}}\left[b_{2} \frac{d^{2}}{d s^{2}}+b_{1} \frac{d}{d s}+b_{0}\right],
$$

where $b_{2}=\left\langle h_{n}(u)\left|a_{02}\right| h_{n}(u)\right\rangle_{u}$,

$$
b_{1}=\left\langle h_{n}(u)\left|a_{11} \frac{\partial}{\partial u}+a_{01}\right| h_{n}(u)\right\rangle_{u}=\left\langle h_{n}(u)\left|a_{01}-\frac{1}{2} \frac{\partial a_{11}}{\partial u}\right| h_{n}(u)\right\rangle_{u},
$$

and

$$
\begin{aligned}
b_{0}+\frac{2 m^{*} E_{2 \mathrm{D}}}{\hbar^{2}} & =\left\langle h_{n}(u)\left|a_{20} \frac{\partial^{2}}{\partial u^{2}}+a_{10} \frac{\partial}{\partial u}+a_{00}\right| h_{n}(u)\right\rangle_{u} \\
& =\left\langle h_{n}(u)\left|a_{00}-(n \pi)^{2} a_{20}-\frac{1}{2} \frac{\partial a_{10}}{\partial u}\right| h_{n}(u)\right\rangle_{u} .
\end{aligned}
$$

Also, by taking into account the periodic boundary conditions for $g_{n}(s)$, the variation $\delta g_{n}(s)$ satisfies $\delta g_{n}(L)=\delta g_{n}(0)$ and $\dot{\delta} g_{n}(L)=\dot{\delta} g_{n}(0)$.

The operator $\Omega_{n}$ is Hermitian since it can be written as

$$
\Omega_{n}=\frac{\hbar^{2}}{2 m^{*}}\left[-\frac{d}{d s} c_{2} \frac{d}{d s}-\frac{i}{2}\left(\frac{d}{d s} c_{1}+c_{1} \frac{d}{d s}\right)+c_{0}\right],
$$

where $c_{2}=-b_{2}$, 


$$
c_{1}=i\left(b_{1}-\frac{d b_{2}}{d s}\right)=\frac{1}{\lambda^{2}}\left\langle h_{n}(u)\left|\frac{u w(1+\eta)+2 \dot{\sigma}}{\eta^{2}}\right| h_{n}(u)\right\rangle_{u},
$$

and

$$
\begin{aligned}
c_{0}= & b_{0}+\frac{i}{2} \frac{d c_{1}}{d s}=\frac{n^{2} \pi^{2}}{w^{2}}-\frac{2 m^{*} E_{2 \mathrm{D}}}{\hbar^{2}}+\left\langle h_{n}(u)\right|-\frac{k^{2}}{4 \eta^{2}}+\frac{3 \dot{w}^{2}}{4 w^{2} \eta^{2}} \\
& +\frac{u w \ddot{k}}{2 \eta^{3}}+\frac{u k \ddot{w}}{\eta^{3}}+\frac{u \dot{k} \dot{w}}{\eta^{3}}-\frac{3 u k \dot{w}^{2}}{w \eta^{3}}-\frac{5 u^{2} w^{2} \dot{k}^{2}}{4 \eta^{4}}-\frac{3 u^{2} k w \dot{k} \dot{w}}{\eta^{4}} \\
& +\frac{n^{2} \pi^{2} u^{2} \dot{w}^{2}}{w^{2} \eta^{2}}+\left(\frac{u w(1+\eta)+2 \dot{\sigma}}{2 \eta \lambda^{2}}\right)^{2}\left|h_{n}(u)\right\rangle_{u} .
\end{aligned}
$$

The Hermitian character of $\Omega_{n}$ guarantees that

$$
\delta L_{n}=2 \operatorname{Re}\left[\left\langle\delta g_{n}(s)\left|\Omega_{n}\right| g_{n}(s)\right\rangle_{s}\right] .
$$

Therefore, the condition $\delta L_{n}=0$ leads to $\Omega_{n} g_{n}(s)=0$, i.e.,

$$
\begin{aligned}
& {\left[-\frac{d}{d s} c_{2} \frac{d}{d s}-\frac{i}{2}\left(\frac{d}{d s} c_{1}+c_{1} \frac{d}{d s}\right)+v_{n}+\beta_{n}\right] g_{n}(s)} \\
& =\frac{2 m^{*} E_{1 \mathrm{D}}}{\hbar^{2}} g_{n}(s)
\end{aligned}
$$

where

$$
E_{1 \mathrm{D}}=E_{2 \mathrm{D}}-\frac{\hbar^{2} n^{2} \pi^{2}}{2 m^{*} \bar{w}^{2}}
$$

is called the longitudinal energy. It is the difference between the two-dimensional energy $E_{2 \mathrm{D}}$ and the mean transversal energy along the quantum ring. The value $\bar{w}$ is the root mean inverse square width, i.e.,

$$
\frac{1}{\bar{w}^{2}}=\frac{1}{L} \int_{0}^{L} \frac{1}{w^{2}(s)} d s .
$$

To express the other coefficients, it is convenient to define the following auxiliary function:

$$
I_{p, q}(n, \alpha)=\left\langle h_{n}(u)\left|\frac{u^{p}}{\eta^{q}}\right| h_{n}(u)\right\rangle_{u}=\int_{-1 / 2}^{1 / 2} \frac{u^{p} h_{n}^{2}(u)}{(1+\alpha u)^{q}} d u .
$$

The coefficient $c_{2}=I_{0,2}(n, \alpha)$ affects the longitudinal effective mass,

$$
c_{1}=\frac{w\left[I_{1,1}(n, \alpha)+I_{1,2}(n, \alpha)\right]+2 \dot{\sigma} c_{2}}{\lambda^{2}}
$$

is related to the threading magnetic flux,

$$
\begin{aligned}
v_{n}= & n^{2} \pi^{2}\left(\frac{1}{w^{2}}-\frac{1}{\bar{w}^{2}}\right)+\left(-\frac{k^{2}}{4}+\frac{3 \dot{w}^{2}}{4 w^{2}}\right) c_{2}+\left(\frac{w \ddot{k}}{2}+k \ddot{w}+\dot{k} \dot{w}\right. \\
& \left.-\frac{3 k \dot{w}^{2}}{w}\right) I_{1,3}(n, \alpha)-\left(\frac{5 w^{2} \dot{k}^{2}}{4}+3 k w \dot{k} \dot{w}\right) I_{2,4}(n, \alpha) \\
& +\frac{n^{2} \pi^{2} \dot{w}^{2}}{w^{2}} I_{2,2}(n, \alpha),
\end{aligned}
$$

is the effective longitudinal potential,${ }^{34}$ and

$$
\begin{aligned}
\beta_{n}= & \frac{1}{\lambda^{4}}\left\{\dot{\sigma}^{2} c_{2}+w \dot{\sigma}\left[I_{1,1}(n, \alpha)+I_{1,2}(n, \alpha)\right]+\frac{w^{2}}{4}\left[I_{2,0}(n)\right.\right. \\
& \left.\left.+2 I_{2,1}(n, \alpha)+I_{2,2}(n, \alpha)\right]\right\}
\end{aligned}
$$

contains the terms leading to the quadratic dependence of the energy levels on the magnetic field strength. It is worth remarking that $I_{2,0}(n)=1 / 12-1 /\left(2 n^{2} \pi^{2}\right)$ and $I_{2,0}(1) \approx 0.0327$.

The solutions of Eq. (36) are written as

$$
g_{n}(s)=\sum_{q} C_{n, q} \zeta_{q}(s),
$$

where the basis functions are

$$
\zeta_{q}(s)=\frac{e^{2 \pi i q s / L}}{\sqrt{L}},
$$

and the coefficients satisfy the following eigenvalue problem:

$$
\sum_{q^{\prime}} M_{q, q^{\prime}}^{(n)} C_{n, q^{\prime}}=E_{1 \mathrm{D}} C_{n, q} .
$$

The matrix elements are given by

$$
\frac{2 m^{*}}{\hbar^{2}} M_{q, q^{\prime}}^{(n)}=\left\langle\zeta_{q}\left|\frac{4 \pi^{2} q q^{\prime} c_{2}}{L^{2}}+\frac{\pi\left(q+q^{\prime}\right) c_{1}}{L}+v_{n}+\beta_{n}\right| \zeta_{q^{\prime}}\right\rangle_{s} .
$$

The numerical results in Sec. VI are calculated with $q$ $=-100,-99, \ldots, 100$, i.e., 201 Fourier terms in Eq. (43).

\section{PERIODICITY OF THE ENERGY LEVELS}

The longitudinal mode $g_{n}(s)$ may be written as

$$
g_{n}(s)=\widetilde{g}_{n}(s) e^{-i \xi(s)},
$$

where

$$
\xi(s)=\frac{e}{\hbar} \int_{0}^{s} \boldsymbol{A}(u, \bar{s}) \cdot \boldsymbol{T}_{c}(\bar{s}) d \bar{s}=\frac{\sigma(s)}{\lambda^{2}} .
$$

Hence, the boundary conditions $g_{n}(L)=g_{n}(0)$ and $\dot{g}_{n}(L)$ $=\dot{g}_{n}(0)$ lead to $\widetilde{g}_{n}(L)=e^{i \gamma \widetilde{g}_{n}}(0)$ and $\dot{\tilde{g}}_{n}(L)=e^{i \gamma} \dot{\widetilde{g}}_{n}(0)$, where

$$
\gamma=\xi(L)=\frac{\sigma(L)}{\lambda^{2}}=\frac{S}{\lambda^{2}}=\frac{2 \pi \Phi}{\Phi_{0}} .
$$

When Eq. (47) is set into Eq. (36), we obtain

$$
\begin{aligned}
& {\left[-\frac{d}{d s} c_{2} \frac{d}{d s}-\frac{i}{2}\left(\frac{d}{d s} \widetilde{c}_{1}+\widetilde{c}_{1} \frac{d}{d s}\right)+v_{n}+\widetilde{\beta}_{n}\right] \widetilde{g}_{n}(s)} \\
& \quad=\frac{2 m^{*} E_{1 \mathrm{D}}}{\hbar^{2}} \widetilde{g}_{n}(s),
\end{aligned}
$$

where

$$
\widetilde{c}_{1}=\frac{w\left[I_{1,1}(n, \alpha)+I_{1,2}(n, \alpha)\right]}{\lambda^{2}}
$$

and 


$$
\widetilde{\beta}_{n}=\frac{w^{2}}{4 \lambda^{4}}\left[I_{2,0}(n)+2 I_{2,1}(n, \alpha)+I_{2,2}(n, \alpha)\right] .
$$

At this point, we note that the energy levels are invariant under translations and rotations of the ring. Moreover, for $|\alpha| \ll 1$, we obtain $c_{2} \approx 1$,

$$
\widetilde{c}_{1} \approx-\frac{3 w \alpha I_{2,0}(n)}{\lambda^{2}}, \quad \text { and } \quad \tilde{\beta}_{n} \approx \frac{w^{2}}{\lambda^{4}} I_{2,0}(n) .
$$

This means that for very thin quantum rings, the coefficients $\widetilde{c}_{1}$ and $\widetilde{\beta}_{n}$ are negligible. In this regime, $\widetilde{g}_{n}(s)$ satisfies

$$
\left[-\frac{d^{2}}{d s^{2}}+v_{n}(s)\right] \widetilde{g}_{n}(s)=\frac{2 m^{*} E_{1 \mathrm{D}}}{\hbar^{2}} \widetilde{g}_{n}(s),
$$

and the only dependence of the energy levels on the magnetic flux comes from the factor $\exp \left(2 \pi i \Phi / \Phi_{0}\right)$ in the boundary conditions for $\widetilde{g}_{n}(s)$. Of course, this factor is periodic in $\Phi$ with a period $\Phi_{0}$. As pointed out by Pershin and Piermarocchi, ${ }^{28}$ such a periodicity is associated with the Aharonov-Bohm oscillations of the energy levels.

\section{COMPENSATION BETWEEN WIDTH AND CURVATURE}

Regarding Eq. (54), the function $v_{n}(s)$ often produces energy gaps, which reduce the amplitude of oscillation of the energy levels. However, when $w$ and $k$ have small first- and second-order derivatives, we obtain

$$
v_{n}(s) \approx n^{2} \pi^{2}\left(\frac{1}{w^{2}(s)}-\frac{1}{\bar{w}^{2}}\right)-\frac{k^{2}(s)}{4} .
$$

Hence, the main effects of a non-uniform width and curvature are compensated for each other, provided $v_{n}(s)$ is a constant. Such a constant is the following mean value:

$$
\bar{v}_{n}=\frac{1}{L} \int_{0}^{L} v_{n}(s) d s=-\frac{\bar{k}^{2}}{4},
$$

where $\bar{k}$ is the root mean square of $k(s)$. This leads to the following width profile:

$$
w(s)=\frac{1}{\sqrt{\frac{1}{\bar{w}^{2}}+\frac{k^{2}(s)-\bar{k}^{2}}{4 \pi^{2} n^{2}}}} .
$$

In this sense, the ring should be thinner when the absolute value of the curvature of the centerline is larger. ${ }^{13}$

\section{NUMERICAL RESULTS}

\section{A. Rings with uniform width}

Here, we study quantum rings with a uniform width $w$ $=15 \mathrm{~nm}$ and limit ourselves to the transversal mode with $n$ $=1$ and energy $\hbar^{2} \pi^{2} /\left(2 m^{*} w^{2}\right) \approx 24.94 \mathrm{meV}$. The longitudinal energy $E_{1}$ will be given in units of $E_{0}=\hbar^{2} \pi^{2} /\left(2 m^{*} L^{2}\right)$. Hence, for a better comparison of the spectra of the different rings considered here, the perimeter of the centerline is taken as $L=942.5 \mathrm{~nm}$ in all of the cases. This corresponds to a
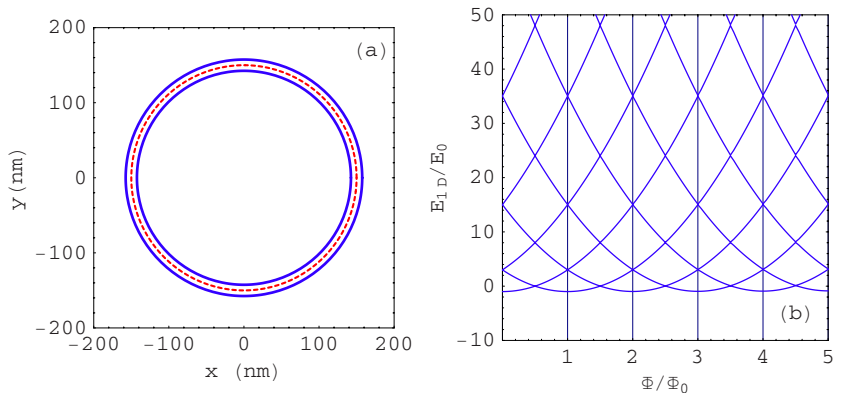

FIG. 1. (Color online) (a) Scheme of a circular ring and (b) energy levels as a function of the magnetic flux. The ring width is $w=15 \mathrm{~nm}$ and the radius of the centerline, which is marked by the dashed line in (a), is $150 \mathrm{~nm}$.

circle with a radius of $150 \mathrm{~nm}$. For conduction electrons in GaAs rings of this size, $E_{0} \approx 0.63 \mathrm{meV}$.

With a fixed perimeter, the area $S=\sigma(L)$ enclosed by the centerline depends on its shape. Numerical results are displayed for energy levels as a function of the magnetic flux $\Phi$ in the range $0 \leq \Phi / \Phi_{0} \leq 5$.

We first consider a circular ring whose centerline has a radius of $150 \mathrm{~nm}$, as shown in Fig. 1(a). Since the area enclosed by the centerline is $S \approx 0.071 \mu \mathrm{m}^{2}$, a threading flux quantum corresponds to a magnetic field of $58.5 \mathrm{mT}$. The typical Aharonov-Bohm oscillations of the energy levels, with a period $\Phi_{0}$, are clearly observed in Fig. 1(b).

The case of an elliptical quantum ring is depicted in Fig. 2(a). Its centerline is an ellipse with semiaxes $a=194.4 \mathrm{~nm}$ and $b=97.5 \mathrm{~nm}$ and encloses the area $S \approx 0.060 \mu \mathrm{m}^{2}$. Hence, a threading flux quantum corresponds to a magnetic field of $69.4 \mathrm{mT}$. Aharonov-Bohm oscillations of the energy levels are also observed in Fig. 2(b). As expected, the nonuniform curvature of the ellipse leads to the occurrence of gaps in the energy spectrum. However, the levels are degenerate when the flux is an odd integer times $\Phi_{0} / 2$. This degeneracy is due to the twofold rotational symmetry of the ring. $^{22}$

A distorted quantum ring similar to those studied by Pershin and Piermarocchi ${ }^{28}$ is also considered. The situation simulates a structural defect in the form of a dip, which may occur in fabricated, grown, or field-effect quantum rings. ${ }^{3,6}$ Instead of a piecewise circular ring, we deal with a centerline
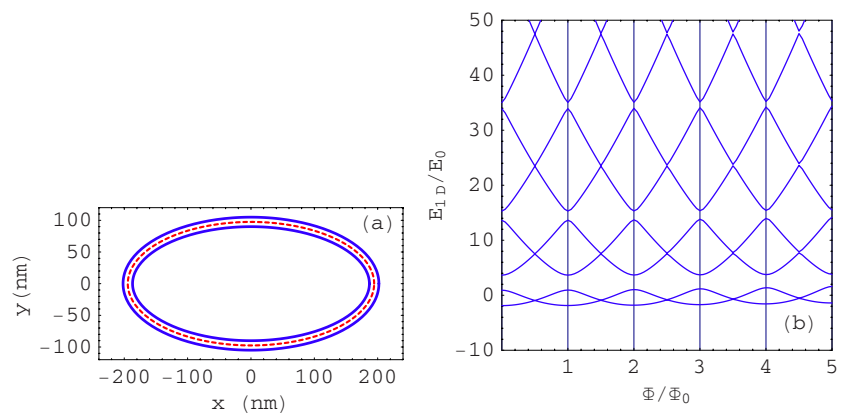

FIG. 2. (Color online) (a) Scheme of an elliptical ring with a width $w=15 \mathrm{~nm}$ and (b) energy levels as a function of the magnetic flux. The semiaxes of the centerline are $a=194.4 \mathrm{~nm}$ and $b$ $=97.5 \mathrm{~nm}$. 

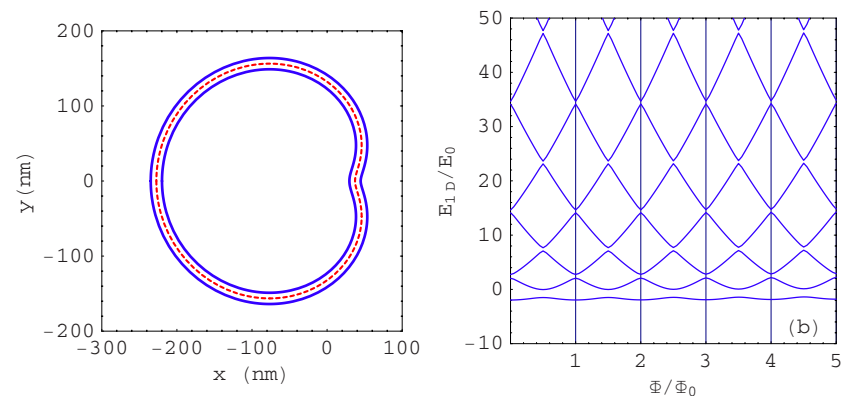

FIG. 3. (Color online) (a) Scheme of a limaçon-shaped ring with a width $w=15 \mathrm{~nm}$ and (b) energy levels as a function of the magnetic flux. The intersections of the centerline with the $x$ axis are $x_{1} \approx-227.4 \mathrm{~nm}$ and $x_{2} \approx 37.2 \mathrm{~nm}$.

in the shape of a limaçon. Such a curve is given in polar coordinates $(\rho, \theta)$ by

$$
\rho=c\left(1-\frac{23}{32} \cos (\theta)\right),
$$

with $c=132.3 \mathrm{~nm}$. In this case, a threading flux quantum corresponds to $B \approx 59.8 \mathrm{mT}$ because the area enclosed by the limaçon is $S \approx 0.069 \mu \mathrm{m}^{2}$. Aharonov-Bohm oscillations of the energy levels are clearly observed in Fig. 3(b), although small gaps are apparent and the ground level is rather flat. This is in good qualitative agreement with Fig. 2(a) of Ref. 28 (the units of energy differ by a factor of 4). Of course, since both rings lack rotational symmetry, we do not expect degenerate energies to occur in either case. ${ }^{9}$

The reason why we did not apply our theory to the distorted ring in Ref. 28 is that Eq. (41) contains $\dot{k}, \dot{k}^{2}$, and $\ddot{k}$. Therefore, the curvature of such a ring is discontinuous and strong singularities occur in Eq. (36). Moreover, since $f(u, s)$ is assumed to be continuous, the wave function $\Psi(x, y)$ in Eq. (15) would be discontinuous. To avoid such complications, we have used the limaçon-shaped centerline, wherein all derivatives of the curvature are continuous.

\section{B. Rings with non-uniform width}

As a simple case of a quantum ring with a non-uniform width, we consider a circular ring with radius of $100 \mathrm{~nm}$ and $w(s)=[20-0.6 \cos (2 \pi s / L)] \mathrm{nm}$. Note that the width increases as $x$ decreases, but this is not apparent in Fig. 4(a) because the amplitude of the oscillation is only $3 \%$ of the mean width. Since the area of the circle is $S \approx 0.126 \mu \mathrm{m}^{2}$, a threading flux quantum occurs for $B \approx 32.9 \mathrm{mT}$. AharonovBohm oscillations of the energy levels, with period $\Phi_{0}$, are observed in Fig. 4(b). However, the non-uniformity of the width produces gaps and a flattening of the lower six energy levels.

The ring in Fig. 4(a) is an accurate approximation of an eccentric ring with circular boundaries with radii of 90 and $110 \mathrm{~nm}$ and eccentricity $\Delta=0.6 \mathrm{~nm}$. Such a ring was studied in Ref. 21, and Fig. 4(b) herein is in excellent agreement with the Fig. 4(b) therein. It should be noted that a very small value in eccentricity has produced a quenching of the Aharonov-Bohm oscillations of the lower energy levels.
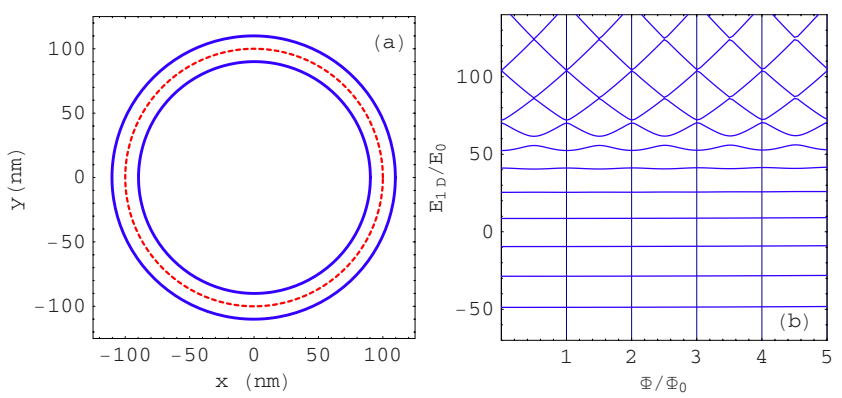

FIG. 4. (Color online) (a) Scheme of a circular ring with a radius of $100 \mathrm{~nm}$ and a width $w(s)=[20-0.6 \cos (2 \pi s / L)] \mathrm{nm}$ and (b) energy levels as a function of the magnetic flux.

This is relevant for experimental studies of persistent currents in quantum rings. However, a more realistic model of the confining potential may be needed to describe the experimental results.

The physical reason for the flattening of the energy levels can be explained by using Eq. (55). The wider regions of the circular ring are more favorable for the electrons, i.e., they correspond to a lower effective potential. Therefore, lowenergy electrons are confined near the point corresponding to $s=L / 2$, wherein the width attains its maximum value. Since the ring is thin, small variations in the width produce large variations in the transversal energy, thus confining the electron. By expanding the longitudinal potential up to second order in the arc length $s-L / 2$, one obtains

$$
\frac{\hbar^{2}}{2 m^{*}} v_{n}(s) \approx-\frac{\hbar^{2} n^{2} \pi^{2}}{2 m^{*}} \frac{\ddot{w}(L / 2)}{w^{3}(L / 2)}\left(s-\frac{L}{2}\right)^{2}+C,
$$

where $C$ is nearly constant. The first term corresponds to an effective one-dimensional oscillator with the following level spacing:

$$
\Delta E_{n}=\frac{\hbar^{2} n \pi}{m^{*}} \sqrt{-\frac{\ddot{w}(L / 2)}{w^{3}(L / 2)}} .
$$

Such a term describes the dominant interaction for weak and moderate magnetic fields and explains the flatness and spacing of the lower energy levels displayed in Fig. 4(b). Namely, in units of $E_{0}=\hbar^{2} \pi^{2} /\left(2 m^{*} L^{2}\right)$, the level spacing is

$$
\frac{\Delta E_{n}}{E_{0}}=\frac{2 n L^{2}}{\pi} \sqrt{-\frac{\ddot{w}(L / 2)}{w^{3}(L / 2)}} .
$$

For the ring in Fig. 4, we have $w(s)=\bar{w}-\Delta \cos (2 \pi s / L)$, with $\bar{w}=100 \mathrm{~nm}$ and $\Delta=0.6 \mathrm{~nm}$. This leads to

$$
\frac{\Delta E_{1}}{E_{0}}=\frac{4 L}{\bar{w}+\Delta} \sqrt{\frac{\Delta}{\bar{w}+\Delta}} \approx 20.8
$$

and $\Delta E_{1} \approx 0.3 \mathrm{meV}$. These numerical results are in good agreement with Fig. 4(b) in this paper and Fig. 4 in Ref. 21, respectively.

Next, we consider a ring with a non-uniform width, with the same centerline as the one shown in Fig. 2(a), i.e., an ellipse with semiaxes $a=194.4 \mathrm{~nm}$ and $b=97.5 \mathrm{~nm}$. In this case, the width profile is displayed in Fig. 5(a). It has been 

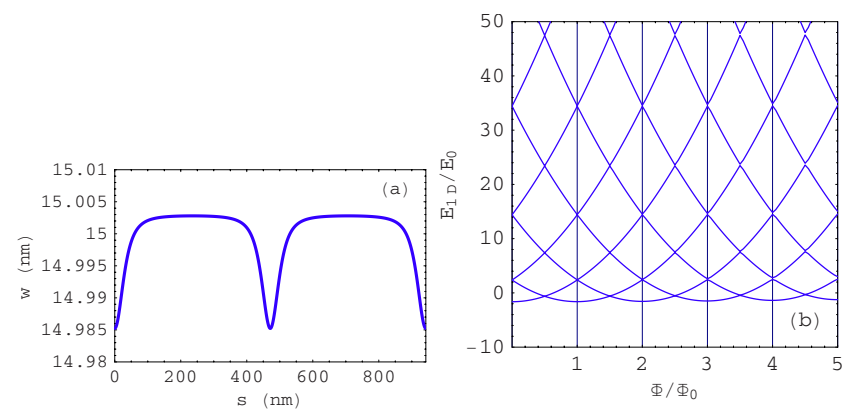

FIG. 5. (Color online) (a) Width profile that compensates for the main effects of the non-uniform curvature of an elliptical ring and (b) energy levels as a function of the magnetic flux. The root mean square width of the ring is $w=15 \mathrm{~nm}$ and the semiaxes of the centerline are $a=194.4 \mathrm{~nm}$ and $b=97.5 \mathrm{~nm}$.

calculated by using Eq. (57) in order to compensate for the main effects of the width and the curvature. The resulting ring is thinner where the centerline has a larger curvature, i.e., at the principal vertices of the ellipse. As expected, gapless Aharonov-Bohm oscillations of the energy levels are apparent in Fig. 5(b).

It is worth noticing that the width variations shown in Fig. 5(a) are very small. In fact, the energy gaps displayed in Fig. 2(b) have been eliminated by variations in the width that are less than $1 \AA$. On the one hand, one must bear in mind that this is an idealized situation. In practice, it is not a feasible task to control the width of a quantum ring with such a high accuracy. However, in some cases, a partial compensation between curvature and width might be attained. On the other hand, measurable variations in the width should noticeably affect the electronic states, thus hiding the interesting effects of the non-uniform curvature. This situation is more dramatic for thinner rings, which are the focus of this work. Of course, a more realistic model with finite-barrier transversal confinement should be developed for a comparison to experimental data. In the same direction, a multichannel approach may also be needed. ${ }^{29}$ Furthermore, uncontrolled interface roughnesses and impurities should be taken into account.

\section{CONCLUSIONS}

We have calculated the electronic states of quantum rings in a threading magnetic field, focusing our attention on the combined effects of the width non-uniformity and the curva- ture profile of the centerline. An effective one-dimensional equation was obtained through a variational separation of variables in curvilinear coordinates. Such an equation contains the width and curvature profiles and their derivatives up to second order. Therefore, it may be helpful for the theoretical analysis and the experimental design of quantum rings. We dealt with thin rings with an arbitrary centerline and a non-uniform width and presented numerical results for circular, elliptical, and limaçon-shaped quantum rings.

Regarding quantum rings with a non-uniform width, we have obtained interesting results. For a circular ring, we proved that smooth and tiny variations in the width may produce quenching of the Aharonov-Bohm oscillations of the lower energy levels. Moreover, a width profile that compensates for the main effects of the curvature variations was obtained for an elliptical centerline. The ring should be thinner where the centerline has a larger curvature. However, for the considered elliptical ring, the accuracy of the required width profile is beyond the technological capabilities. Therefore, the experimental observation of the compensation effect is not a feasible task. Nonetheless, this means that uncontrolled variations in the width of the ring should probably dominate over the curvature profile.

Our theory, which deals with electronic states in rings with a non-uniform width in a very intuitive and numerically efficient manner, should be relevant for the quantum mechanics in curved spaces ${ }^{23-25}$ and for the physics of quantum rings. We have shown that variations in the width can compensate for or dominate over the effects of a non-uniform curvature, thus affecting the manifestation of the AharonovBohm effect. Furthermore, we suspect that the width nonuniformity may play a similar role in electronic and matter transmissions through curved waveguides. ${ }^{30-33}$

The use of Dirichlet boundary conditions in our simple model may overestimate the effects of the width variations on the electronic spectra of quantum rings. Anyway, our predictions should encourage the investigation of more realistic models with finite-barrier transversal confinement ${ }^{28}$ and interchannel coupling. ${ }^{29}$ Additionally, the present formalism paves the way for those purposes.

\section{ACKNOWLEDGMENTS}

The authors are grateful to the Brazilian agencies FAPESP and CNPq for financial support. A.B.-A. thanks T. A. de Oliveira for useful discussions.

\footnotetext{
*alexys@fc.unesp.br

†latge@if.uff.br

${ }^{1}$ S. M. Reimann and M. Manninen, Rev. Mod. Phys. 74, 1283 (2002).

${ }^{2}$ W.-C. Tan and J. C. Inkson, Phys. Rev. B 60, 5626 (1999).

${ }^{3}$ G. E. Philipp, J. A. M. Galeana, C. Cassou, P. D. Wang, C. Guasch, B. Vögele, M. C. Holland, and C. M. Sotomayor Torres, in Diagnostic Techniques for Semiconductor Materials Processing II, edited by S. W. Pang, O. J. Glembocki, F. H. Pollak, F.
}

Celli, and C. M. Sotomayor Torres, MRS Symposia Proceedings No. 406 (Materials Research Society, Pittsburgh, 1996), p. 307.

${ }^{4}$ S. Pedersen, A. E. Hansen, A. Kristensen, C. B. Sørensen, and P. E. Lindelof, Phys. Rev. B 61, 5457 (2000).

${ }^{5}$ A. Fuhrer, S. Lüscher, T. Ihn, T. Heinzel, K. Ensslin, W. Wegscheider, and M. Bichler, Nature (London) 822, 413 (2001).

${ }^{6}$ T. Raz, D. Ritter, and G. Bahir, Appl. Phys. Lett. 82, 1706 (2003).

${ }^{7}$ T. Mano et al., Nano Lett. 5, 425 (2005). 
${ }^{8}$ T. Kuroda, T. Mano, T. Ochiai, S. Sanguinetti, K. Sakoda, G. Kido, and N. Koguchi, Phys. Rev. B 72, 205301 (2005).

${ }^{9}$ N. A. J. M. Kleemans et al., Phys. Rev. Lett. 99, 146808 (2007).

${ }^{10}$ Z. Barticevic, M. Pacheco, and A. Latgé, Phys. Rev. B 62, 6963 (2000).

${ }^{11}$ J. Splettstoesser, M. Governale, and U. Zülicke, Phys. Rev. B 68, 165341 (2003).

${ }^{12}$ J. Simonin, C. R. Proetto, Z. Barticevic, and G. Fuster, Phys. Rev. B 70, 205305 (2004).

${ }^{13}$ A. Bruno-Alfonso and T. A. de Oliveira, Braz. J. Phys. 36, 434 (2006).

${ }^{14}$ J. Planelles, J. I. Climente, and F. Rajadell, Physica E (Amsterdam) 33, 370 (2006).

${ }^{15}$ M. Aichinger, S. A. Chin, E. Krotscheck, and E. Räsänen, Phys. Rev. B 73, 195310 (2006).

${ }^{16}$ V. M. Fomin, V. N. Gladilin, S. N. Klimin, J. T. Devreese, N. A. J. M. Kleemans, and P. M. Koenraad, Phys. Rev. B 76, 235320 (2007).

${ }^{17}$ M. Amado, R. P. A. Lima, C. González-Santander, and F. Domínguez-Adame, Phys. Rev. B 76, 073312 (2007).

${ }^{18}$ L. I. Magarill, D. A. Romanov, and A. V. Chaplik, JETP 83, 361 (1996).

${ }^{19}$ D. Berman, O. Entin-Wohlman, and M. Y. Azbel, Phys. Rev. B 42, 9299 (1990).

${ }^{20}$ L. A. Lavenère-Wanderley, A. Bruno-Alfonso, and A. Latgé, J. Phys.: Condens. Matter 14, 259 (2002).
${ }^{21}$ A. Bruno-Alfonso and A. Latgé, Phys. Rev. B 71, 125312 (2005).

22 J. Planelles, F. Rajadell, and J. I. Climente, Nanotechnology 18, 375402 (2007).

${ }^{23}$ R. C. T. da Costa, Phys. Rev. A 23, 1982 (1981).

${ }^{24}$ P. Exner and P. Seba, J. Math. Phys. 30, 2574 (1989).

${ }^{25}$ G. Dunne and R. L. Jaffe, Ann. Phys. 223, 180 (1993).

${ }^{26}$ I. J. Clark and A. J. Bracken, J. Phys. A 29, 339 (1996).

${ }^{27}$ D. Gridin, A. T. I. Adamou, and R. V. Craster, Phys. Rev. B 69, 155317 (2004).

${ }^{28}$ Y. V. Pershin and C. Piermarocchi, Phys. Rev. B 72, 125348 (2005).

${ }^{29}$ L. Wendler, V. M. Fomin, and A. A. Krokhin, Phys. Rev. B 50, 4642 (1994).

${ }^{30}$ S. N. Shevchenko and Y. A. Kolesnichenko, J. Exp. Theor. Phys. 92, 811 (2001).

${ }^{31}$ O. Olendski and L. Mikhailovska, Phys. Rev. B 66, 035331 (2002).

${ }^{32}$ Y. V. Pershin and C. Piermarocchi, Phys. Rev. B 72, 195340 (2005).

${ }^{33}$ M. Jääskeläinen and S. Stenholm, Phys. Rev. A 66, 043612 (2002).

${ }^{34}$ This is in agreement with Eq. (5) and Eqs. (8)-(13) of Ref. 13, except for a mistype in Eq. (12) therein. The coefficient 3 within the brackets in the latter equation should have been written outside instead. The mistype did not affect the numerical results. 